\title{
Serotonin challenge normalized deficits in executive planning in patients with obsessive-compulsive disorder (OCD)
}

\author{
Christine Lochner $^{1}$, Samuel R. Chamberlain², Martin Kidd³ ${ }^{3}$ Lian Taljaard¹, Dan J. Stein ${ }^{1,4}$ \\ ${ }^{1}$ MRC Unit on Risk and Resilience in Mental Disorders, Department of Psychiatry, Stellenbosch University, South Africa \\ ${ }^{2}$ Department of Psychiatry, University of Cambridge; and Peterborough NHS Foundation Trust, Cambridge, UK \\ ${ }^{3}$ Centre for Statistical Consultation, Department of Statistics and Actuarial Sciences, University of Stellenbosch, South Africa \\ ${ }^{4}$ Department of Psychiatry, University of Cape Town, South Africa
}

\section{Background}

* Problems with executive planning and response inhibition are common in OCD. ${ }^{1,2}$

* Substantial evidence supports the efficacy of selective serotonin reuptake inhibitors (SSRIs) in OCD. ${ }^{3}$

* Yet the potential role of serotonin in these OCD-related cognitive deficits is unclear.

* Pharmacological challenge is recognized as a means of interrogating brain neurochemical systems and their associated cognitive functions.

* Therefore, this study aimed to address the potential of serotonin challenge for altering specific executive functions in OCD patients, first-degree relatives of OCD patients \& healthy controls.

\section{Materials and Methods}

* A randomized double-blind crossover study design was utilised to assess the effects of a single dose of the SSRI escitalopram (20mg) and placebo on specific executive functions in 24 adults with OCD $(60.7 \% \mathrm{~F}), 13$ clinically unaffected 1 st-degree OCD relatives $(84.6 \% \mathrm{~F})$ and 28 healthy controls $(54.2 \% \mathrm{~F})$.

* Performance on the One-touch Stockings of Cambridge (OTS; to measure executive planning) and Stop-Signal Task (SST; to measure response inhibition), respectively, were assessed 3-4 hours after oral administration of the pharmacological challenge/placebo, using a mixed model analysis of covariance (ANCOVA).
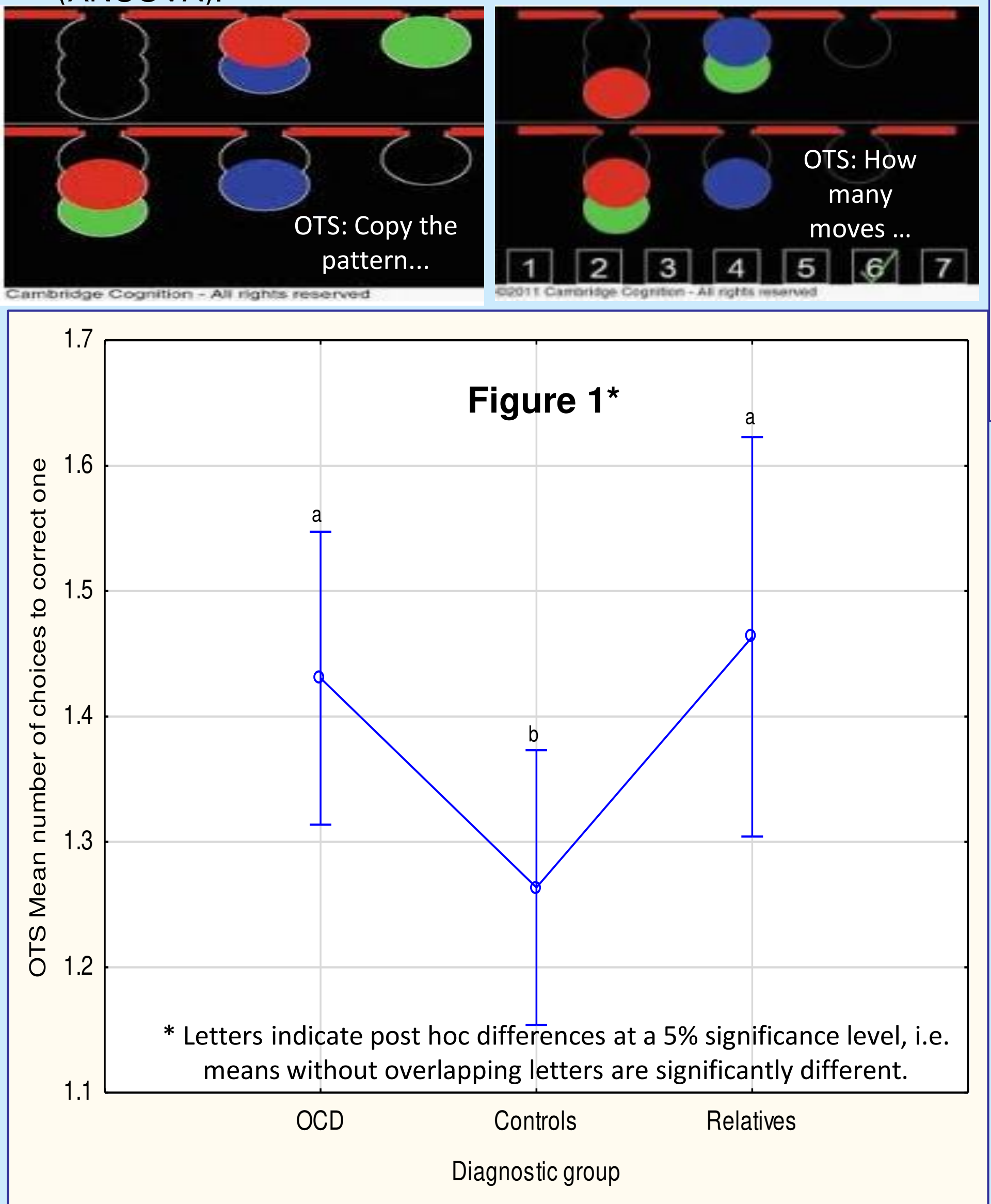

\section{Results}

* On the executive planning task (OTS), there was a significant effect of group (Fig. $1 ; \mathrm{F}=3.1 ; \mathrm{p}=0.05$ ) and a trend towards a significant group $\mathrm{x}$ treatment condition interaction (Fig. 2; $F=2.8, p=0.07$ ).

* Post hoc tests showed: (i) that patients and their relatives were impaired on the task overall compared to controls $(p=0.04, p=0.03)$; and (ii) that escitalopram was associated with significantly improved executive planning in the OCD group $(p=0.01)$, but not in the other two groups (both $p>0.1)$ (Fig. 2).

- No main effect of group or group x treatment interactions were observed for response inhibition (SST; both $p>0.1$ ).

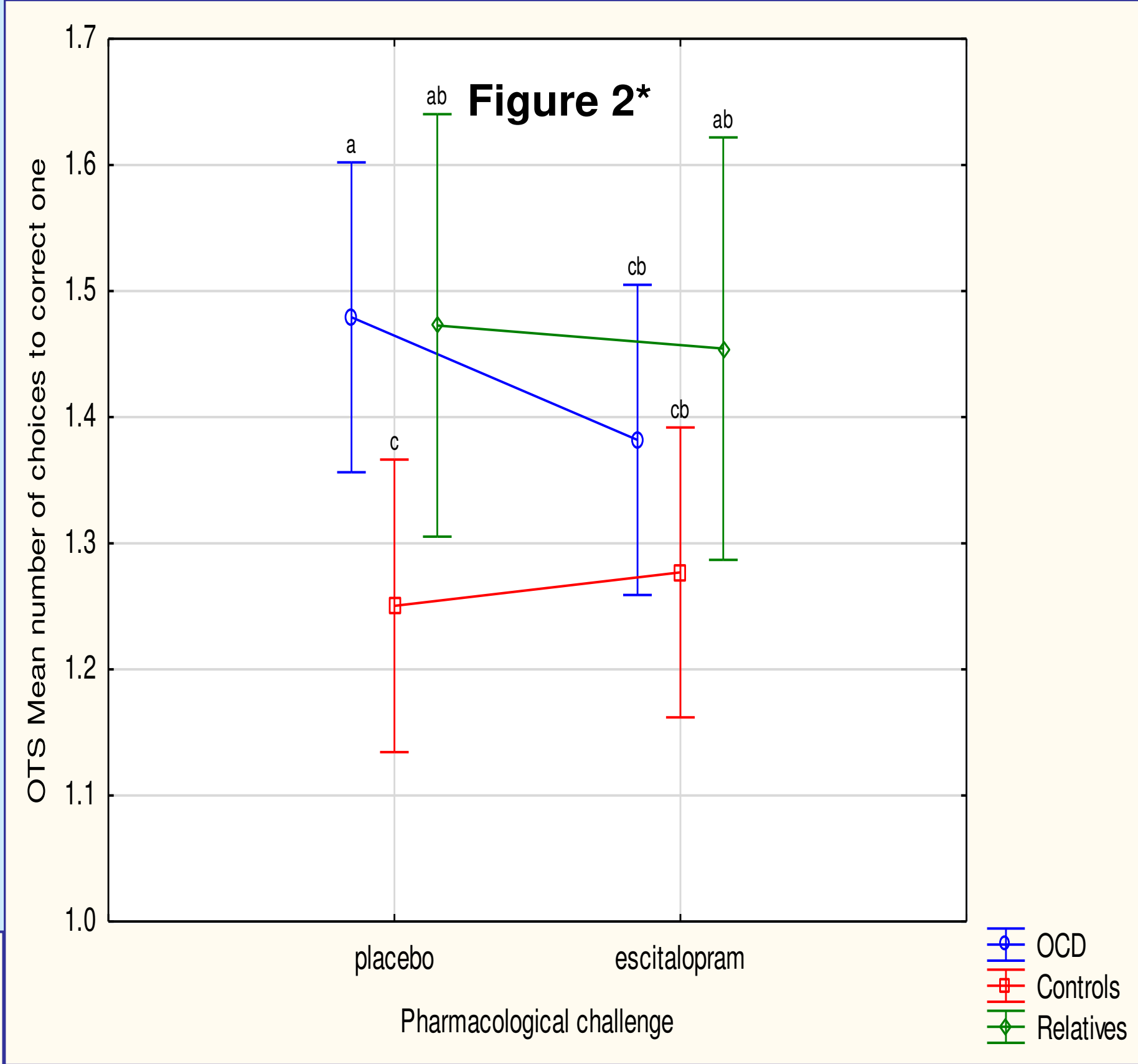

\section{Conclusions}

Our findings indicated that executive planning did not differ between OCD patients and OCD relatives while the differences between these two groups and controls were significant. Acute administration of escitalopram normalized this executive planning dysfunction in OCD, providing support for the role SSRIs may play in the normalization of planning deficits of the fronto-striatal circuitry typical of OCD.

This effect was not significant in the relatives of patients; this could be attributable to lower statistical power, or may suggest differential effects of serotonin challenge in people with current OCD symptoms.

Lack of effects of escitalopram on response inhibition is in keeping with a role for other neurochemical systems in this function, notably noradrenaline, as found in previous non-OCD work.

Acknowledgements: The Medical Research Council of South Africa, the Obsessive-Compulsive Foundation (DJS), the National Research Foundation of South Africa (CL), and an unrestricted grant from Lundbeck H/S. SRC's role in this project was funded by a Wellcome Trust Clinical Fellowship. 